\title{
Doxorubicin-loaded dextran-based nano-carriers for highly efficient inhibition of lymphoma cell growth and synchronous reduction of cardiac toxicity
}

This article was published in the following Dove Press journal:

International Journal of Nanomedicine

\section{Ying Fang, ${ }^{1}, *$ Hao Wang, ${ }^{2, *}$ Hong-Jing Dou, ${ }^{2, *}$ Xing Fan, ${ }^{1}, *$ Xiao-Chun Fei, ${ }^{3}$ Lei Wang, ${ }^{2}$ Shu Cheng,' Anne Janin, ${ }^{4,5}$ Li Wang, ${ }^{1,4}$ Wei-Li Zhao ${ }^{1,4}$}

'State Key Laboratory of Medical Genomics, Shanghai Institute of Hematology, Shanghai Rui Jin Hospital, Shanghai Jiao Tong University School of Medicine, Shanghai, China; ${ }^{2}$ State Key Laboratory of Metal Matrix Composites, School of Materials Science and Engineering, Shanghai Jiao Tong University, Shanghai, China; ${ }^{3}$ Department of Pathology, Shanghai Rui Jin Hospital, Shanghai Jiao Tong University School of Medicine, Shanghai, China; ${ }^{4}$ SinoFrench Research Center of Life Science and Genomics, Laboratory of Molecular Pathology, Shanghai, China; 5Joint Research Unit II65, Inserm, University Paris VII, Saint-Louis Hospital, Paris, France

*These authors contributed equally to this work

Correspondence: Wei-Li Zhao; Li Wang State Key Laboratory of Medical Genomics, Shanghai Institute of Hematology, Shanghai Rui Jin Hospital, 197 Rui Jin Er Road, Shanghai 200025,

China

Tel +862164370045

Fax +862164743206

Email zhao.weili@yahoo.com; wl_wangdong@l26.com
Purpose: Cardiac side effects of doxorubicin (Dox) have limited its clinical application. The aim of this study was to explore new Dox-loaded dextran-based nano-carriers (NCs) in efficiently targeting tumor growth with less cardiac toxicity.

Methods: Inspired by recent reports that polymeric NCs could function as sustained, controlled and targeted drug delivery systems, we developed Dox-loaded NCs which displayed a 2-fold release ratio of Dox in the mimic tumor site condition (pH 5.0 with $10 \mathrm{mM}$ glutathione, $\mathrm{GSH}$ ) as much as that in systemic circulation condition ( $\mathrm{pH}$ 7.4).

Results: Lymphoma cells treated with Dox-NCs had significantly higher intracellular Dox concentrations and more apoptotic induction, with lower P-gp expression, when compared with those treated with Dox alone. The identified mechanism of action, apoptosis, was triggered through survivin reduction and caspase-3 activation. Even in the Dox-resistant cells, Dox-NCs could significantly inhibit cell growth and induce apoptosis. In murine lymphoma xenograft models, Dox-NCs also remarkably significantly retarded tumor growth, assessed by murine weight, and demonstrated less cytotoxicity. Noticeably, apoptotic myocardial cells were decreased in the Dox-NCs-treated group, when compared with the control group, which was consistent with low intracellular Dox concentration in the cardiac cell line $\mathrm{H} 9 \mathrm{C} 2$.

Conclusion: Dox-NCs showed an anti-lymphoma effect with reduced cardiac toxicity in both in vivo and in vitro models and, therefore, could be a potential therapeutic agent in the treatment of lymphoma.

Keywords: anti-lymphoma activity, targeted therapy, doxorubicin-loaded, dextran, nanocarrier, cardiac toxicity

\section{Introduction}

Doxorubicin (Dox) is widely used in cancer treatment, however, its clinical application is limited due to its side effects, notably cardiac toxicity. ${ }^{1,2} \mathrm{P}$-gp is a notorious transmembrane bump, critically involved in tumor cell resistance to Dox. ${ }^{3}$ The increasing expressions of P-gp promote tumor cell ability to efflux a wide range of cytotoxic agents, which leads to treatment failure. ${ }^{4}$

Polymeric nano-carriers (NCs) have been reported as promising carriers of many anticancer drugs, ${ }^{5,6}$ due to their function as sustained, controlled and targeted drug delivery systems. ${ }^{7-10}$ By modifying the size and the surface charge, we designed a type of dextran-based nanogel with high biocompatibility and biosafety, which can be retained for a long time in lymph nodes. ${ }^{11}$ The aim of this study was to develop Dox-loaded 
NCs, which selectively enter the lymphatic system and efficiently eliminate malignant lymphocytes. The carrier size, zeta potential, surface charge, morphology, drug loading content, encapsulation efficiency, cellular uptake and cytotoxicity to both lymphoma cells and normal cells, as well as P-gp expression, were investigated in vitro. In vivo, the anti-lymphoma effects of these new Dox-loaded NCs were also evaluated on xenograft mouse models.

\section{Materials and methods Materials}

Dextran (molecular weight $[\mathrm{Mw}]=40,000 \mathrm{Da}$ ), ceric ammonium nitrate, and methyl acrylate were purchased from Shanghai Sinopharm Chemical Reagent Co., Ltd. (Huangpu District, Shanghai, China) and purified as described previously. ${ }^{12}$ Diallyl disulfide (Sigma Aldrich, St Louis, MO, USA) was used after vacuum distillation. Doxorubicin hydrochloride (Dox) was purchased from Beijing Zhong Shuo Pharmaceutical Technology Development Co., Ltd. (Dongcheng, Beijing, China).

\section{Preparation}

Dox-NCs were fabricated through self-assembly assisted polymerization approach, as previously developed. ${ }^{11-13}$ Two steps were included in the self-assembly assisted polymerization approach. The first step was the fabrication of dextranbased NCs bearing redox sensitive disulfide crosslinking junctions via a highly efficient self-assembly assisted approach developed by our group. Specifically, $2.5 \mathrm{~g}$ of dextran was fully dissolved in $50 \mathrm{~mL}$ of water and kept under $\mathrm{N}_{2}$ protection at $30^{\circ} \mathrm{C}$. Then initiator ceric ammonium nitrate ( $1.208 \mathrm{~g}$ dissolved in $1.25 \mathrm{~mL}$ of $0.1 \mathrm{M} \mathrm{HNO}_{3}$ ) was added, and 5 minutes later the monomer, methyl acrylate $(1.34 \mathrm{~mL})$, was added into the solution. After 30 minutes, the cross-linker diallyl disulfide ( $0.244 \mathrm{~mL}$ dissolved in $1 \mathrm{~mL}$ of DMSO) was added into the system. The entire process was allowed to proceed for 4 hours and dialyzed against deionized water for 3 days. Finally, the solution was freeze-dried to obtain NCs. The second step involved Dox being covalently conjugated to NCs via a hydrazone bond through the same method as previously reported. ${ }^{12}$

\section{Characterization}

Hydrodynamic diameter and zeta potential were measured using a Zetasizer Nano ZS90 instrument (Malvern Panalytical, Malvern, UK). Transmission electron microscopy (TEM) images were characterized using a JEM-2100 (JEOL Ltd., Akishima, Tokyo, Japan) instrument. ${ }^{1} \mathrm{H}$ NMR spectra were recorded by an Avance III $400 \mathrm{MHz}$ spectrometer (Bruker,
Faellanden, Switzerland). The Dox content and in vitro drug release was determined via the UV-Vis absorbance method using a Shimadzu UV-2550 UV-Vis spectrophotometer (Shimadzu, Tokyo, Japan) (the concentration of Dox-NCs was $0.5 \mathrm{mg} / \mathrm{mL}) .{ }^{12}$

\section{Cells and reagents}

Human lymphoma cell lines, Jurkat, H9, Namalwa, and SUDHL-4, as well as a rat cardiac cell line H9C2 were purchased from American Type Culture Collection (ATCC, Manassas, VA, USA) and cultured according to the manufacturer's recommendations. In order to achieve hypoxic conditions, cells were cultured in a hypoxia chamber with $1 \%$ oxygen. Dox-resistant cells were created by continuously exposing the cells to increasing concentrations of Dox, as previously reported. ${ }^{14}$

\section{Cell viability}

Cell viability was assessed using a Cell Counting Kit-8 (Dojindo Laboratories, Tokyo, Japan), and absorbance was measured at $450 \mathrm{~nm}$ by spectrophotometry.

\section{ATP assay}

The ATP assay was performed as previously described. ${ }^{15}$ Cells were incubated either with Dox or with Dox-NCs, for 24 hours and 48 hours. Intracellular ATP levels were detected using an ATP assay kit (Beyotime Biotechnology, Jiangsu, China); cells were lysed using the lysis buffer and centrifuged at $12,000 \times g$ for 5 minutes at $4^{\circ} \mathrm{C}$. Fifty microliters of supernatant was mixed immediately with $100 \mu \mathrm{L}$ of dilution buffer containing luciferase which had been preincubated at room temperature for 3 minutes. Luminance (RLU) was determined by using a Luminometer (Berthold Technologies, Bad Wildbad, Germany). The concentration of ATP was calculated using a previously determined standard curve and normalized using the cellular protein level.

\section{Flow cytometry}

Cell apoptosis was assessed using the Annexin V-FITC Apoptosis Kit (Becton Dickinson, Franklin Lakes, NJ, USA) and performed according to the manufacturer's instructions. For detecting the intracellular concentration of Dox, cells were exposed to the indicated concentration of Dox-NCs or Dox, for 10 minutes, 20 minutes, 30 minutes, 1 hour, 2 hours, 3 hours, 6 hours, 12 hours, 24 hours, 36 hours, 48 hours, 60 hours, 72 hours, 84 hours and 96 hours. The concentration of Dox and Dox-NCs was $50 \mathrm{nM}$ for Jurakt and Namalwa cells, and 42 nM for H9 and SU-DHL-4 cells. Then, 
cells were washed with PBS three times, and the intracellular fluorescence of Dox was evaluated by flow cytometry at an excitation of $595 \mathrm{~nm}^{16}$

\section{Terminal deoxytransferase-catalyzed DNA-nick-end labeling (TUNEL) assay}

In situ cell apoptosis was confirmed by detection of fragmented DNA, using the TUNEL assay, using the DeadEnd ${ }^{\mathrm{TM}}$ Colorimetric TUNEL System (Promega Corporation, Madison, WI, USA) according to the manufacturer's instructions. ${ }^{17}$

\section{Western blot}

Cells were lysed in $200 \mu \mathrm{L}$ lysis buffer $(0.5 \mathrm{M}$ Tris- $\mathrm{HCl}$, pH 6.8, 2 mM EDTA, 10\% glycerol, 2\% SDS and 5\% $\beta$-mercaptoethanol). Electrophoresis was performed on $10 \%$ SDS polyacrylamide gels using samples with a concentration of $20 \mu \mathrm{g}$ protein and transferred to nitrocellulose membranes. Membranes were blocked with 5\% non-fat dried milk in Tris-buffered saline and incubated for 2 hours at room temperature with an appropriate primary antibody (caspase-3, cleaved caspase-3, survivin, P-gp), followed by the horseradish peroxidase-conjugated secondary antibody (goat anti-mouse-IgG and goat anti-rabbit-IgG). The immunocomplexes were visualized using the chemiluminescence phototope-horseradish-peroxidase kit (Cell Signaling Technology, Beverly, MA, USA). Actin was used to ensure equivalent protein loading. Horseradish peroxidaseconjugated goat anti-mouse-IgG and goat anti-rabbit-IgG antibodies were purchased from Santa Cruz Biotechnology (Santa Cruz, CA, USA).

\section{Murine model}

Nude mice (5-6-weeks-old) were obtained from Shanghai Laboratory Animal Center and injected subcutaneously with $4 \times 10^{7}$ Jurkat or $7 \times 10^{6}$ Namalwa cells into the right flank. Tumor volumes were calculated as $0.5 \times a \times b,{ }^{2}$ where "a" is the length and " $\mathrm{b}$ " is the width. Treatment was started after the tumor measured $\sim 0.5 \times 0.5$ (length $\times$ width) $\mathrm{cm}$ in surface on Day 0. The mice were divided into treatment groups, consisting of 10 mice per group. The mice in the untreated group were only given RPMI-1640, whereas the mice in the Dox group were administered an intraperitoneal injection of Dox $(6 \mathrm{mg} / \mathrm{kg}$, twice a week) and the mice in the Dox-NCs group were administered an intraperitoneal injection of DoxNCs (6 mg/kg, twice a week), for 2 weeks. The Shanghai Rui Jin Hospital Animal Care and Use Committee approved the experiments, which were conducted in accordance with their approved protocols.

\section{Pathological analysis}

Mice were euthanized at day 14, and subcutaneous tumors and hearts were dissected and cut into two parts. The samples were then prepared in 2 ways; (i) immediately snap frozen; (ii) formaldehyde-fixed and paraffin-embedded for analysis. Immunohistochemistry was performed on $5 \mu \mathrm{m}$-paraffin tumor sections using the indirect immunoperoxidase method and antibodies against Ki67 (DAKO, Glostrup, Denmark) and P-gp. The TUNEL assay was performed on the tumor and heart samples to determine the degree of cellular apoptosis.

\section{Statistical analysis}

All assays were set up in triplicate and the results were expressed as the mean \pm SD of data obtained from three separate experiments. Student's $t$-test was applied to compare two normally distributed groups and Bonferroni to perform multiple comparisons. $P<0.05$ was considered statistically significant. All statistical analyses were evaluated using Statistical Package for the Social Sciences (SPSS) 13.0 software (SPSS Inc., Chicago, IL, USA).

\section{Results \\ Characterization of Dox-NCs}

First, NCs were fabricated using a modified self-assembly assisted polymerization method. Successful preparation of the NCs was verified by an absorption signal at $1,732 \mathrm{~cm}^{-1}$ exhibited by Fourier transform infrared spectroscopy (Figure 1A). This absorption signal corresponded to the ester bonds in the poly(methyl acrylate) chains. The NCs exhibited a uniform spherical shape with a diameter of $\sim 45 \mathrm{~nm}$ under TEM observation (Figure 1B) and a zeta potential of $-3.8 \mathrm{mV}$. In the second step, the NCs were conjugated with Dox, the color of the solution changed from milky white to red, the diameter of NCs increased to $\sim 65 \mathrm{~nm}$ (Figure 1C) and the zeta potential was $-28.5 \mathrm{mV}$. UVabsorbance measurements revealed that the Dox-NCs exhibited a drug loading content of $\sim 11.2 \mathrm{wt} \%$ (Figure 1D). In vitro drug release behavior of Dox-NCs is shown in Figure 1E. The cumulative drug release from the NCs reached $\sim 35 \%$ at a $\mathrm{pH}$ of 5.0 with $10 \mathrm{mM} \mathrm{GSH}$. However, the drug release rate was only $\sim 16 \%$ at a $\mathrm{pH}$ of 7.4 .

\section{Dox-NCs increased intracellular Dox concentration and lymphoma cell cytotoxicity}

To mimic in vivo hypoxia inside the tumor lymphoma, cell lines Jurkat, H9, Namalwa and SU-DHL-4 were cultured under hypoxic conditions and exposed to either Dox-NCs 
A

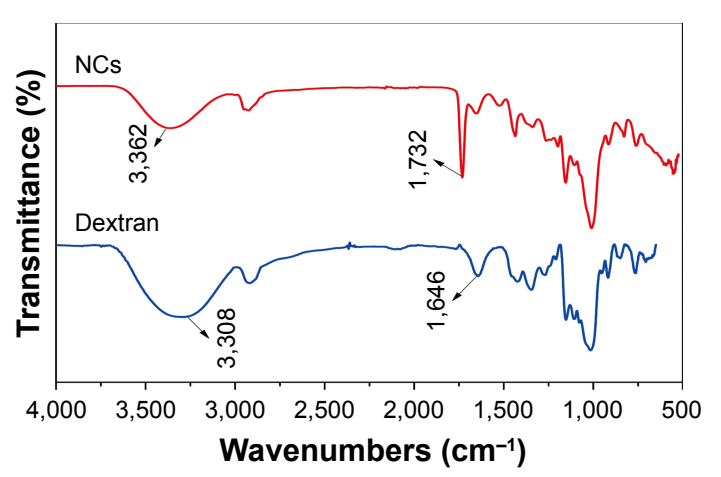

D

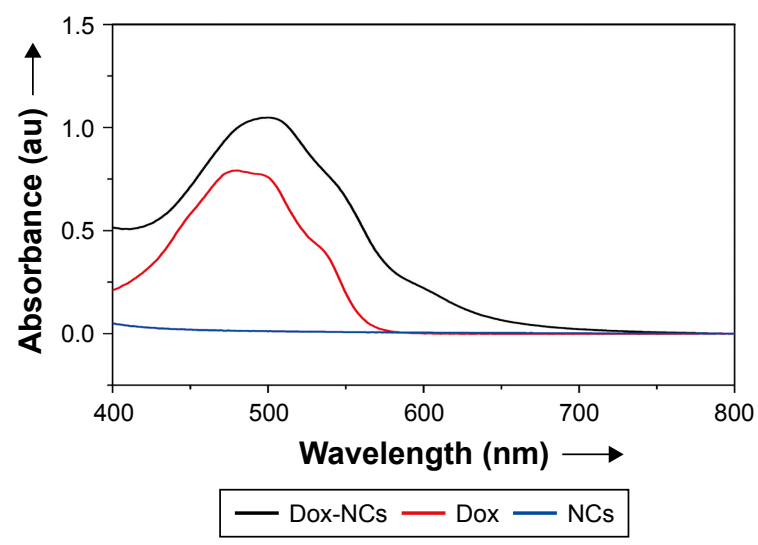

B

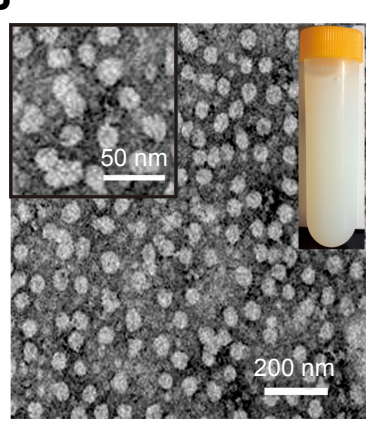

C

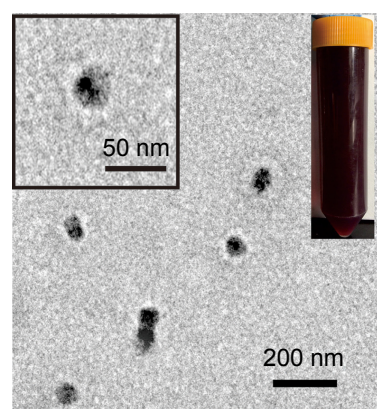

E

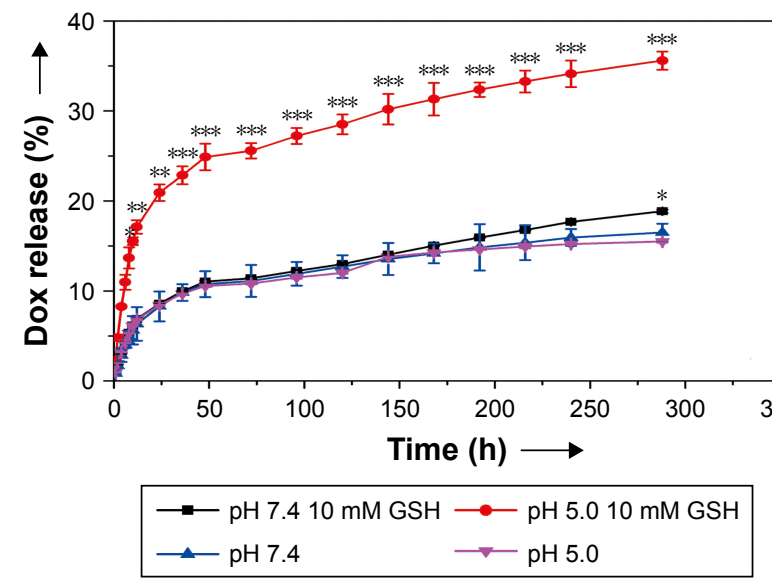

Figure I Features of Dox-NCs.

Notes: (A) FTIR spectra of dextran and NCs. TEM images of NCs (B) and Dox-NCs (C). The insets in (B) and (C) were the photos of NCs in aqueous (40 mg/mL) and DoxNCs solutions ( $3 \mathrm{mg} / \mathrm{mL}$ ), respectively. (D) The UV-Vis absorbance spectra of Dox-NCs, NCs and Dox. The concentration of Dox-NCs was $0.5 \mathrm{mg} / \mathrm{mL}$. The concentration of $\mathrm{NCs}$ was the equivalent concentration to Dox-NCs. The drug loading content of Dox was calculated by the UV-Vis calibration curve of Dox solutions at $480 \mathrm{~nm}$. (E) In vitro drug release profiles of Dox-NCs at a pH of 7.4 with GSH (black curve) or without GSH (blue curve), and at pH =5.0 with GSH (red curve) or without GSH (pink curve). Dox-NCs significantly increased Dox release at a $\mathrm{pH}$ of 5.0 with $10 \mathrm{mM} \mathrm{GSH}$, when compared with that at a $\mathrm{pH}$ of $7.4, * P<0.05, * * P<0.0 \mathrm{I}, * * * P<0.00 \mathrm{I}(\mathrm{n}=3$ ).

Abbreviations: Dox, doxorubicin; FTIR, Fourier transform infrared spectroscopy; GSH, glutathione; NCs, nano-carriers; TEM, transmission electron microscopy.

or Dox for 7 days. Intracellular Dox concentrations were detected at indicated time points. At 36 hours, the fluorescence intensity markedly increased and remained higher in the Dox-NCs-treated cells, when compared with Doxtreated cells (Figure 2A). Additionally, Dox-NCs were more toxic to lymphoma cells, with a significantly lower IC50

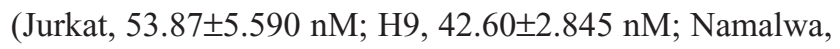

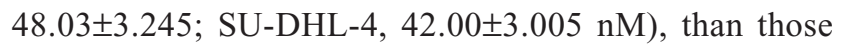
in the Dox treatment group (Jurkat, $72.37 \pm 3.289 \mathrm{nM}, \mathrm{H} 9$, $62.70 \pm 2.663 \mathrm{nM}$, Namalwa, $61.93 \pm 2.338 \mathrm{nM}$, SU-DHL-4, $59.37 \pm 3.560 \mathrm{nM}$, Figure 2B), in consistent with the growth inhibition curves (Figure 2C).

\section{Dox-NCs induced lymphoma cell} apoptosis through downregulating survivin Apoptosis, a type of programmed cell death, is usually used as an indicator of treatment efficacy. As revealed by TUNEL assay and Annexin-V analysis, Dox-NCs markedly increased apoptosis in both Jurkat and Namalwa cells, when compared with Dox treatment (Figure 3A and B). Caspase-3 activation, accompanied by the inhibition of the anti-apoptotic molecule survivin, were observed as the mechanisms of action in the Dox-NC treated cells (Figure 3C).

\section{Dox-NCs inhibited P-gp induction and induced Dox-resistant cell apoptosis}

Accumulating evidence has shown that Dox-treatment could induce tumor cell P-gp expression, resulting in increased drug excretion and multidrug resistance. ${ }^{18,19}$ Compared with Dox, Dox-NCs inhibited P-gp expression (Figure 4A). As the mechanism of action, cytoplasmic ATP concentration was significantly lower, after 48 hours incubation, in Dox-NCs-treated cells (Jurkat $0.63 \pm 0.11 \mathrm{nmol} / 10^{6}$ cells, Namalwa $0.71 \pm 0.29 \mathrm{nmol} / 10^{6}$ cells $)$, when compared with Dox-treated cells (Jurkat $1.34 \pm 0.11 \mathrm{nmol} / 10^{6}$ cells, Namalwa $1.66 \pm 0.22 \mathrm{nmol} / 10^{6}$ cells, $P<0.05$, Figure 4B). Acquired 

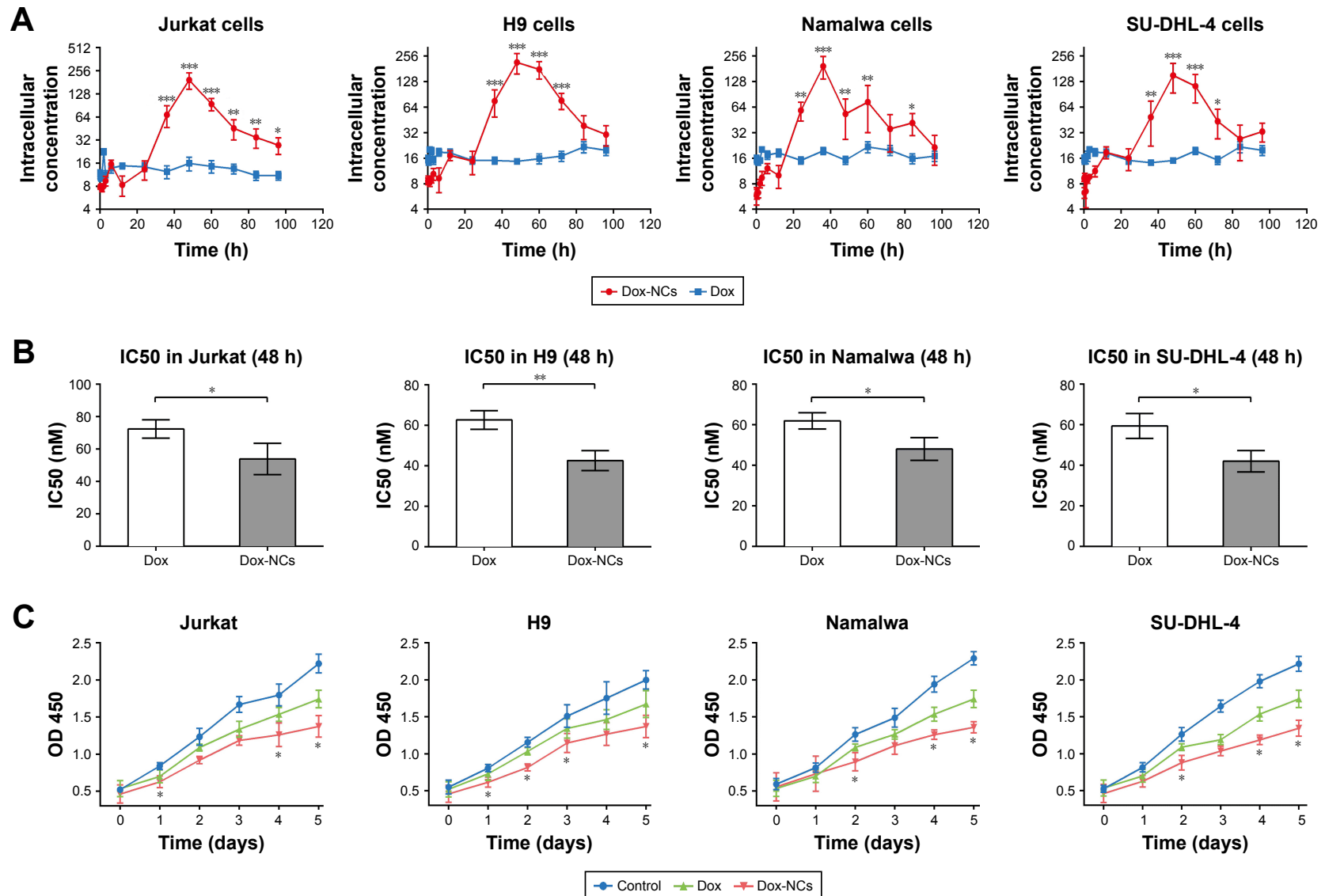

Figure 2 Dox-NCs significantly inhibited lymphoma cell growth.

Notes: (A) Jurkat and Namalwa cells were incubated with $50 \mathrm{nM}$ Dox or Dox-NCs, while H9 and SU-DHL-4 cells were incubated with 42 nM Dox or Dox-NCs for indicated time. Intracellular fluorescence of Dox (red) was evaluated by flow cytometry after excitation at $595 \mathrm{~nm}$. Dox-NCs (red curve) significantly increased intracellular Dox concentration in lymphoma cell lines. ${ }^{*} P<0.05, * * P<0.01$, $* * * P<0.001$ compared with Dox. (B) Cell viability was assessed by the Cell Counting Kit-8 method after 48 hours of exposure to the indicated concentration of Dox and Dox-NPs and IC50 were analyzed. The concentration of Dox and Dox-NCs were $50 \mathrm{nM}$ for Jurkat and Namalwa, $42 \mathrm{nM}$ for $\mathrm{H} 9$ and SU-DHL-4. Each value represents the mean $\pm S D(n=3)$. IC50 of Dox-NCs was significantly lower in lymphoma cell lines than those of $D o x$. $* P<0.05$, $* * P<0.01$. (C) Lymphoma cell lines were treated with Dox and Dox-NCs for the indicated time, the concentrations of Dox and Dox-NCs were $50 \mathrm{nM}$ for Jurkat and Namalwa, $42 \mathrm{nM}$ for H9 and SU-DHL-4, and cell numbers were assessed by the Cell Counting Kit-8 method. The growth curves showed that Dox-NCs significantly inhibited lymphoma cell growth. $* P<0.05$ compared with Dox.

Abbreviations: Dox, doxorubicin; h, hours; NCs, nano-carriers.

drug resistance is an significant obstacle that impairs the success of cancer treatment. Isogenic Dox-resistant sublines were developed as previously reported ${ }^{14,18}$ at a concentration of $15 \mathrm{nM}$ (Jurkat/15 nM Dox and Namalwa/15 nM Dox). Compared with parental cells (Jurkat $72.37 \pm 3.289 \mathrm{nM}$, Namalwa $61.93 \pm 2.338 \mathrm{nM}$ ), the IC50 of Dox significantly increased in Jurkat/15 nM Dox cells $(116.70 \pm 7.46 \mathrm{nM})$ and Namalwa/15 nM Dox cells $(87.73 \pm 5.99 \mathrm{nM})$. Dox-NCs not only decreased the IC50 in both Jurkat/15 nM Dox cells $(76.00 \pm 10.12 \mathrm{nM})$ and Namalwa/15 $\mathrm{nM}$ Dox cells (64.27 $\pm 4.19 \mathrm{nM}$, Figure 4C), but also increased apoptosis of the Dox-resistant cells (Figure 4D).

\section{Dox-NCs exhibited in vivo anti-lymphoma activity with less cardiac toxicity}

The in vivo anti-tumor activity of Dox-NCs was further evaluated in murine xenograft models. Subcutaneous inoculation of Jurkat or Namalwa cells into nude mice resulted in tumor formation at the injection sites of all the mice. The sizes of tumors formed in Dox-NC treated mice were significantly smaller than those of the control group and Dox group after 14 days of treatment $(P<0.0001$, Figure 5A). Consistent with the in vitro study, the expression of P-gp and Ki67 were lower in the Dox-NC-treated tumor cells than those with Dox. (Figure 5B). However, TUNEL assay showed that apoptotic tumor cells were more abundant in the Dox-NCs group than in the Dox group (Figure 5B). On the other hand, the mice in the Dox-NC group demonstrated less weight loss than those in the Dox group; which is indicative of low treatment toxicity with Dox-NCs (Figure 5A, left panel). Cardiac toxicities are the most important adverse effect of Dox. Compared with the Dox group, mice treated with Dox-NCs had less apoptosis of cardiac cells as revealed by hematoxylineosin staining and TUNEL assay (Figure 5C). Further in vitro 

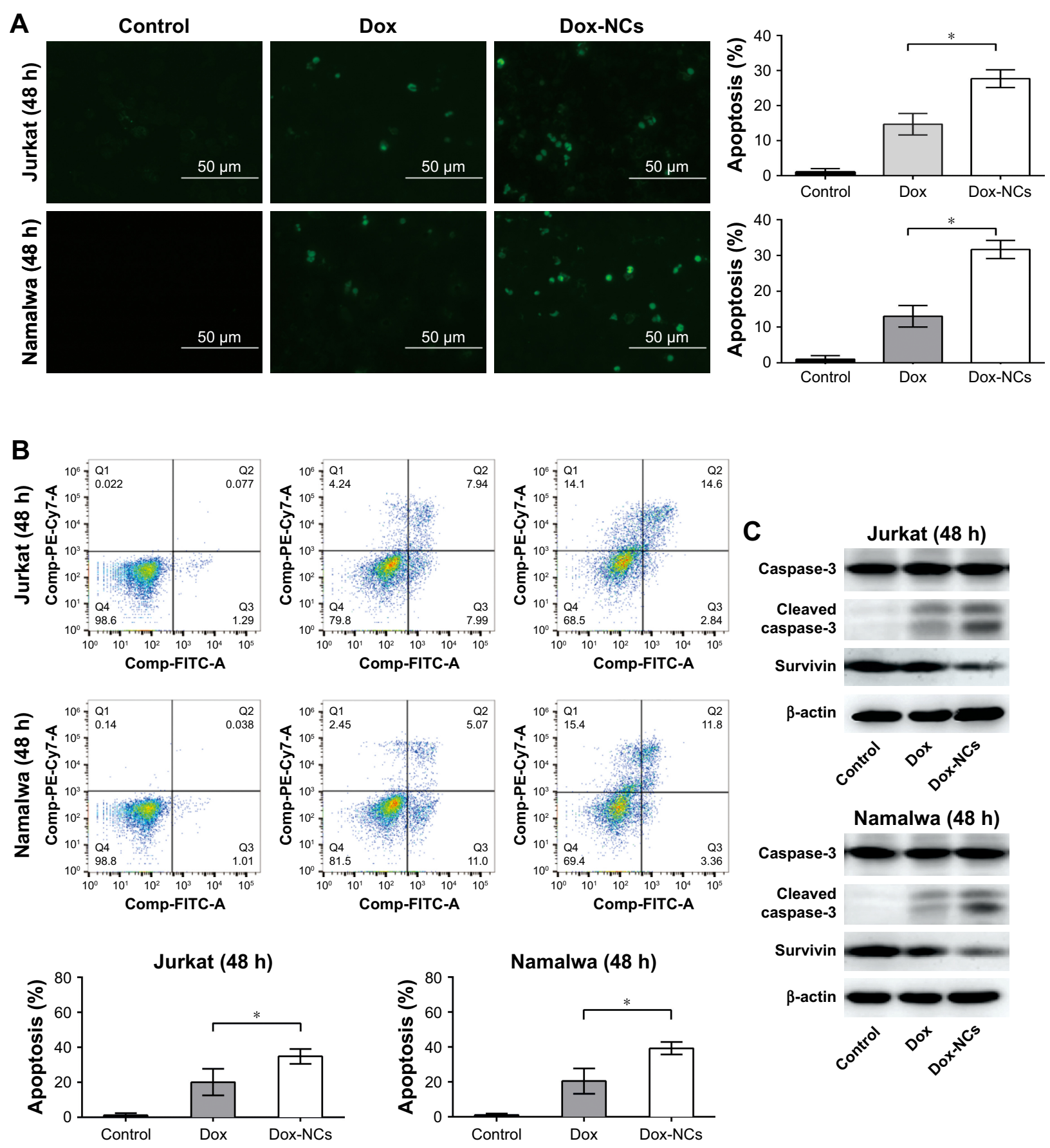

Figure 3 Dox-NCs enhanced lymphoma cell apoptosis through downregulation of survivin and activation of caspase-3.

Notes: Jurkat and Namalwa cells were incubated with $50 \mathrm{nM}$ Dox or Dox-NCs for 48 hours. Dox-NCs enhanced lymphoma cell apoptosis, which was detected by two different methods, TUNEL assay (A) and Annexin $V$ analysis (B). (C) Dox-NCs downregulated survivin expression and activated caspase-3 cleavage in both Jurkat and Namalwa cells after 48 hours treatment. Scale bar set at $50 \mu \mathrm{m}, * P<0.05$.

Abbreviations: Dox, doxorubicin; h, hours; NCs, nano-carriers; TUNEL, terminal deoxytransferase-catalyzed DNA-nick-end labeling assay.

studies showed that the intracellular concentration of Dox was significantly lower in the Dox-NC treated cardiac cell line H9C2, than the Dox-treated cells. In conjunction with the intracellular Dox concentration, reduced apoptotic cells $(11.3 \% \pm 5.6 \%)$ were found with the Dox-NC treated cells, when compared with those treated with Dox $(30.2 \% \pm 4.3 \%$, $P<0.05$, Figure 5D).

\section{Discussion}

To our knowledge, this is the first report evaluating the dualstimulus response of Dox-NCs, which significantly enhanced lymphoma cell growth inhibition, even in Dox-resistant cells. Additionally, Dox-NCs showed less toxicity towards normal cardiac cells. Since Dox-NCs are comprised of both redox sensitive disulfide crosslinking junctions and acid-labile 
A

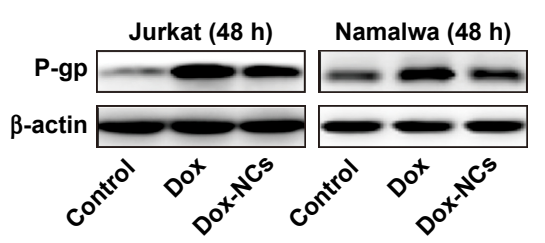

B

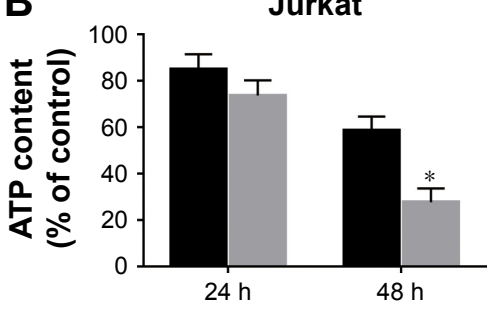

Namalwa

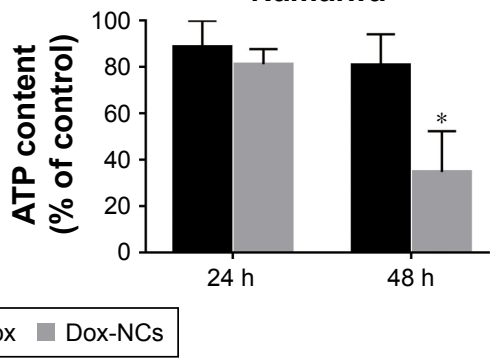

C Jurkat
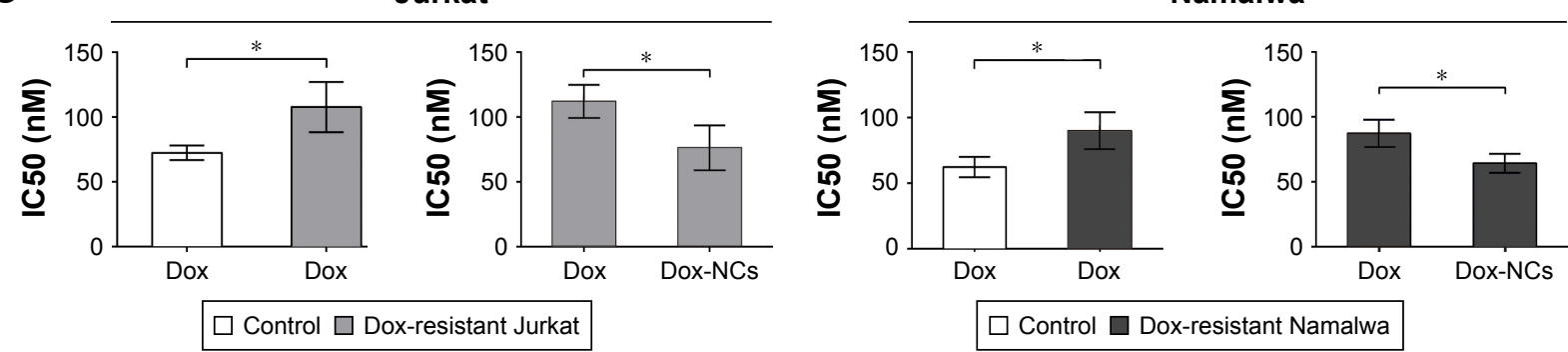

D
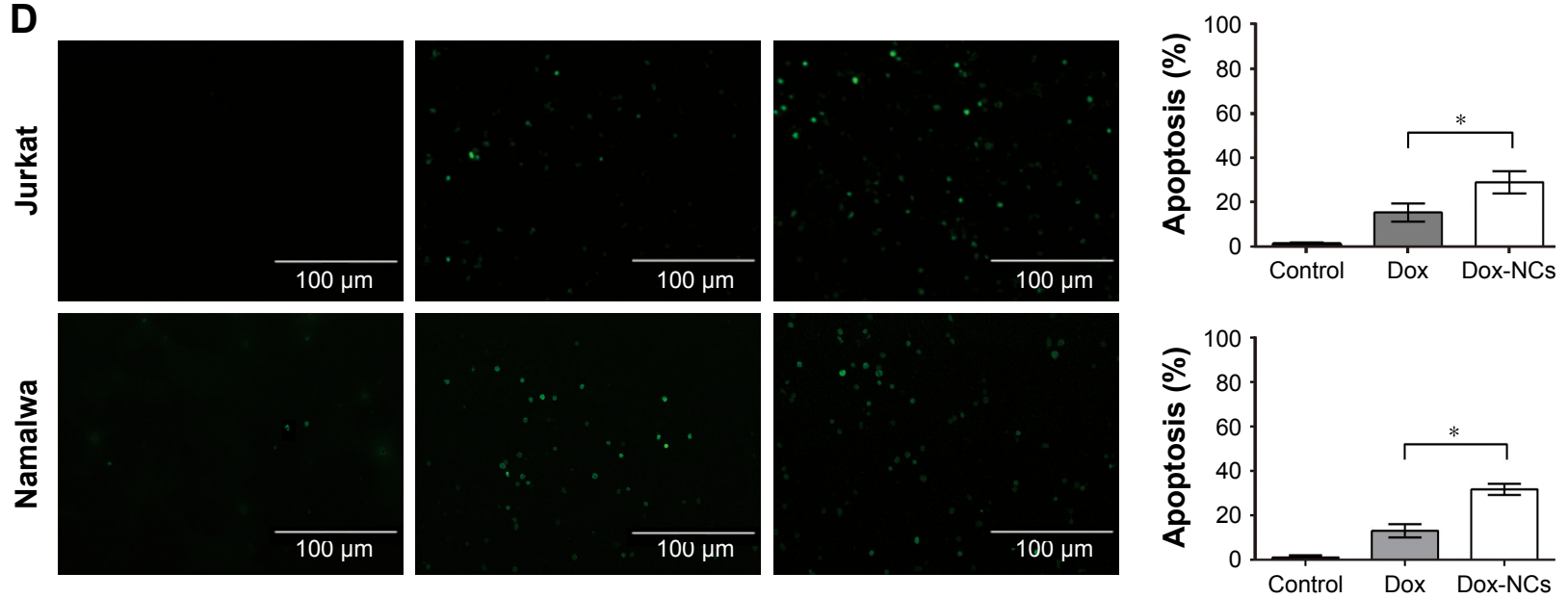

Figure 4 Dox-NCs inhibited Dox-induced P-gp expression and overcome Dox-resistance.

Notes: (A) Dox treatment $(50 \mathrm{nM})$ for 48 hours upregulated P-gp expression in both Jurkat and Namalwa cells. Dox-NCs at the same concentration (50 $\mathrm{nM}$ ) partially inhibited Dox-induced P-gp expression. (B) ATP in cell lysates were detected after 24 hours and 48 hours incubation with 50 nM Dox or Dox-NCs. After 48 hours of treatment, Dox-NCs significantly depleted ATP in both Jurkat and Namalwa cells, $* P<0.05$ compared with Dox. Each value represents the mean \pm SD ( $n=3$ ). (C) IC50 of Dox was significantly increased in Dox-resistant Jurkat and Namalwa cells when compared with their parental cells. IC50 of Dox-NCs was markedly decreased in Dox-resistant cells. $* P<0.05$ comparing with Dox. Each value represents the mean $\pm S D(n=3)$. (D) TUNEL assay showed that Dox-NCs enhanced apoptosis in Dox-resistant Jurkat and Namalwa cells. $* P<0.05$ compared with Dox. Scale bar set at $100 \mu \mathrm{m}$.

Abbreviations: Dox, doxorubicin; h, hours; NCs, nano-carriers; TUNEL, terminal deoxytransferase-catalyzed DNA-nick-end labeling assay.

hydrazone bonds (-28.5 mV), Dox-NCs can display a 2-fold release ratio of the drug in mimicked tumor site conditions (pH 5.0 with $10 \mathrm{mM} \mathrm{GSH}$ ), as well as in the mimicked systemic circulation conditions ( $\mathrm{pH}$ 7.4). This contributed to the accelerated drug release behavior in the mimicked tumor site conditions. The disulfide crosslinking units within NCs were cleaved in a reductive environment and the subsequent hydrazone bond was broken in an acidic environment. ${ }^{20-22} \mathrm{NC}$ size and membrane charge are important factors for the molecules to selectively enter the lymphatic system, ${ }^{23,24}$ which is also known as passive selection. We have constructed optimal dextran-based NCs for lymph node mapping. ${ }^{11}$ Based on this technique, the Dox-NCs, with narrowly dispersed spherical shape $(\sim 65 \mathrm{~nm})$, were successfully prepared. These features enabled Dox-NCs to be passively selected, and retained, in the lymphatic system, which might be a potential option for lymphoma treatment.

Since high glucose uptake is a characteristic of cancer cells, polysaccharide coating can increase cellular uptake. ${ }^{25}$ This may explain why Dox-NCs enter the plasma of lymphoma cells more efficiently than Dox. Moreover, it is reported that modification of the drug loading system not only provokes cytotoxicity but also suppresses P-gp expression. ${ }^{26}$ Here our data also showed that Dox coated 


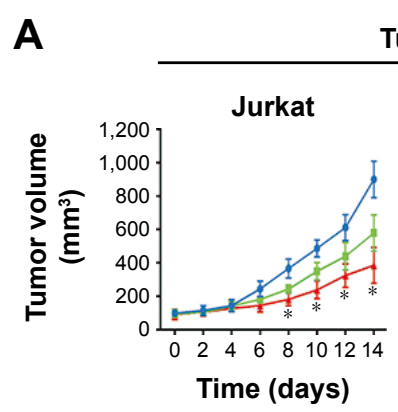

B
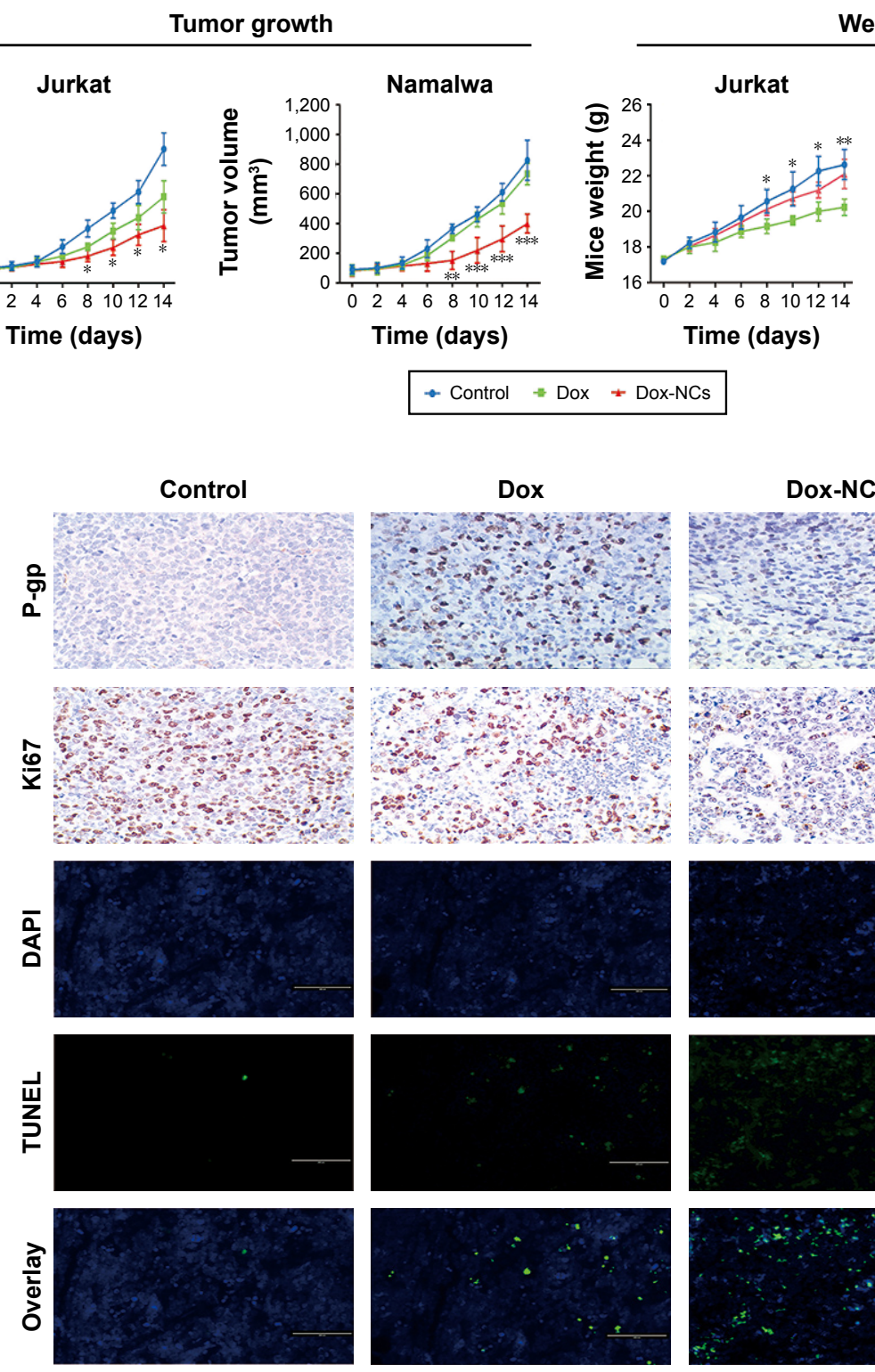

$$
\text { - Control }=\text { Dox } \quad \text { Dox-NCs }
$$

Weight

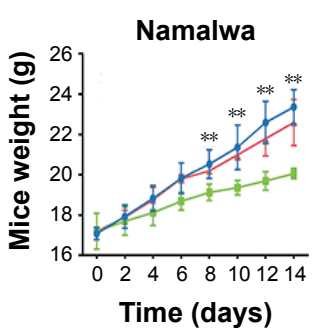




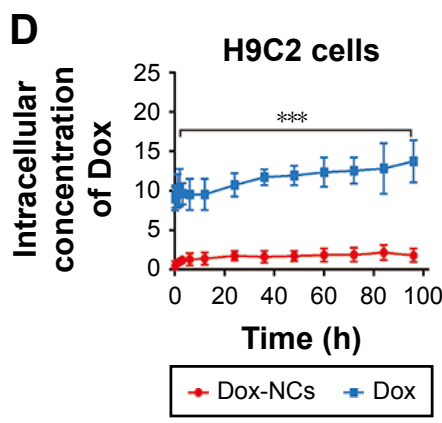

H9C2 cell lines $(48 \mathrm{~h})$
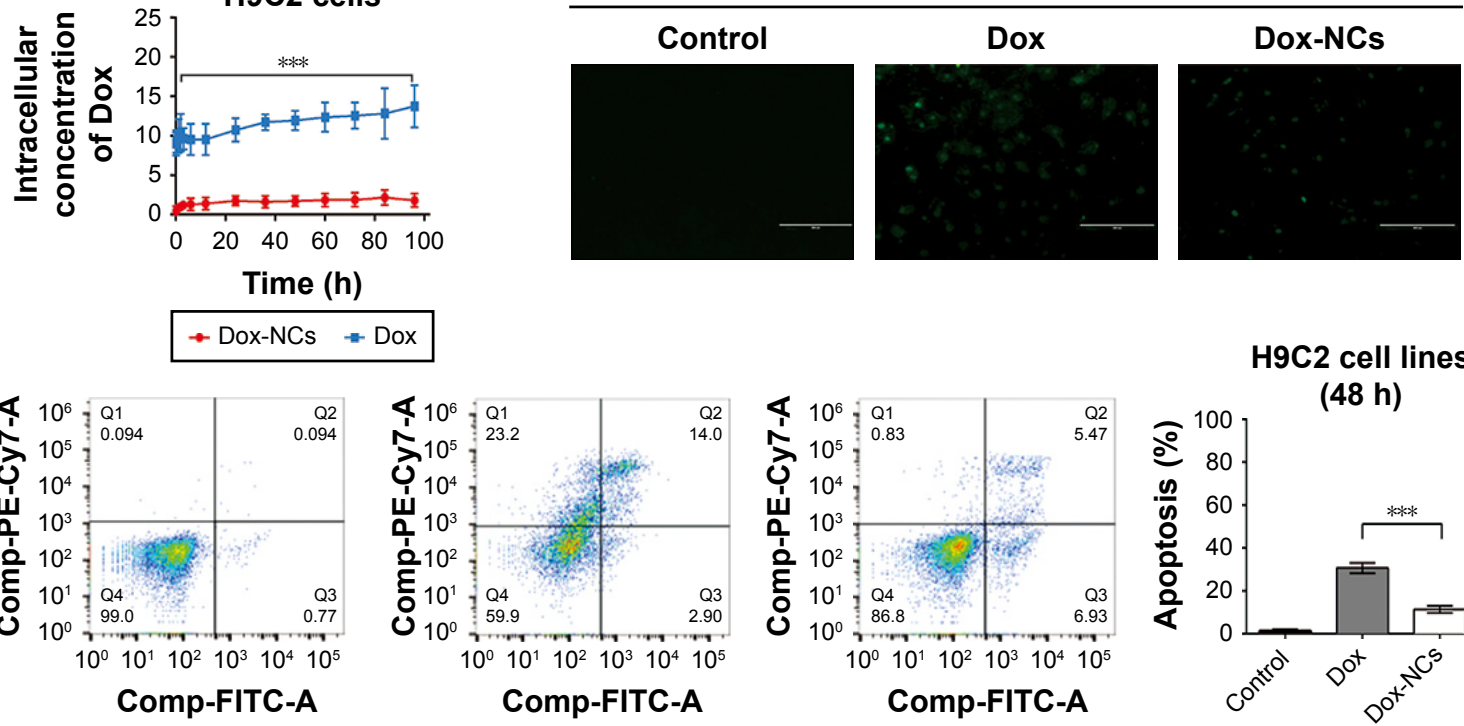

H9C2 cell lines

(48 h)

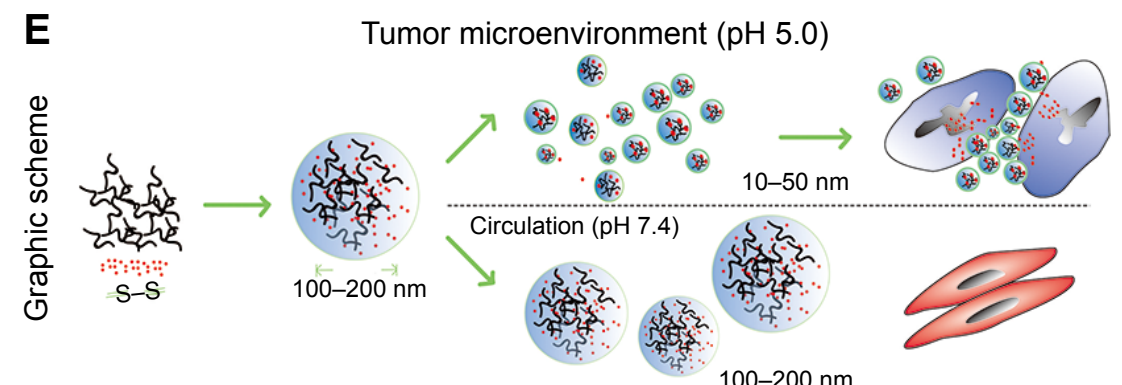

$100-200 \mathrm{~nm}$

\begin{tabular}{|c|c|c|c|}
\hline Doxorubicin & (2) Nano-carriers & (3) Tumor cells & $\Longleftrightarrow$ Cardiac cells \\
\hline
\end{tabular}

Figure 5 In vivo effects of Dox-NCs in murine lymphoma xenograft models.

Notes: (A) In both T- and B-cell lymphoma xenograft models, intraperitoneal treatment of Dox-NCs significantly inhibited tumor growth without affecting the weight of the mice. $* P<0.05$, $* * P<0.0$ I, $* * * P<0.00$ I compared with Dox. (B) As compared with the Dox group, decreased proliferative index Ki67 and low expression of $\mathrm{P}$-pg were observed in the tumors of the Dox-NC group. TUNEL assay showed that Dox-NCs enhanced tumor apoptosis significantly. Scale bar set at I00 $\mu$ m. (C) Hematoxylin-eosin staining and TUNEL assay revealed diminished apoptosis of cardiac cells by Dox-NCs in vivo. Scale bar set as I00 $\mu \mathrm{m}$ (D) Dox-NCs induced cellular apoptosis to a lesser degree in cardiac cell line $\mathrm{H} 9 \mathrm{C} 2$ than Dox. $* * * P<0.000$ I compared with Dox. Scale bar set as $100 \mu \mathrm{m}$. (E) Dox-loaded dextran-based NCs are an efficient drug delivery system to treat malignant lymphoma with less cardiac toxicities.

Abbreviations: Dox, doxorubicin; h, hours; NCs, nano-carriers; TUNEL, terminal deoxytransferase-catalyzed DNA-nick-end labeling assay.

with dextran increased cellular uptake and inhibited P-gp expression, blocking excretion of the drug, which in turn led to increased cellular Dox concentrations. Consequently, the high intracellular concentration of Dox, inhibition of tumor growth and induction of apoptosis were more prominent in Dox-NCs-treated lymphoma cells. Overexpression of P-gp promotes cell resistance to Dox and induces multidrug insensitivity. In the present study, Dox-NCs effectively killed the isogenic Dox-resistant cells with high P-gp expression, which is significant for clinical application.

While being highly toxic to tumor cells, Dox-NCs proved to be a safer treatment option inducing less weight loss in the mice than Dox. In the circulation, toxic Dox was encapsulated by dextran, which is non-toxic to normal cells. A lower cellular uptake of Dox-NCs by cardiac cells resulted in lower toxicity, which is advantageous in that it reduces the side effects on normal organs. ${ }^{27}$

\section{Conclusion}

We concluded that Dox-loaded dextran-based NCs are efficient drug delivery systems to treat malignant lymphoma with less cardiac toxicity.

\section{Acknowledgments}

This study was supported, in part, by research funding from the National Natural Science Foundation of China 
(81830007, 81520108003, 81670716, 81201863 and 21374061), the Shanghai Commission of Science and Technology (14430723400, 14140903100, 14520710300 and 16JC1405800), Shanghai Municipal Education Commission Gao Feng Clinical Medicine Grant Support (20152206, 20152208), Interdisciplinary Program of Shanghai Jiao Tong University (YG2014QN16) and the Chang Jiang Scholars Program.

\section{Author contributions}

WLZ, LW and HJD designed research studies, YF and HW conducted experiments, XF analyzed data, XCF performed pathological analysis, HW, LW fabricated Dox-NCs, and LW, AJ and WLZ wrote the manuscript. All authors contributed toward data analysis, drafting and revising the paper and agree to be accountable for all aspects of the work.

\section{Disclosure}

The authors report no conflicts of interest in this work.

\section{References}

1. Barry E, Alvarez JA, Scully RE, Miller TL, Lipshultz SE. Anthracyclineinduced cardiotoxicity: course, pathophysiology, prevention and management. Expert Opin Pharmacother. 2007;8(8):1039-1058.

2. Lipshultz SE, Colan SD, Gelber RD, Perez-Atayde AR, Sallan SE, Sanders SP. Late cardiac effects of doxorubicin therapy for acute lymphoblastic leukemia in childhood. N Engl J Med. 1991;324(12): 808-815.

3. Ueda K, Cardarelli C, Gottesman MM, Pastan I. Expression of a fulllength cDNA for the human "MDR1" gene confers resistance to colchicine, doxorubicin, and vinblastine. Proc Natl Acad Sci U S A. 1987; 84(9):3004-3008.

4. Fang Z, Chen S, Qin J, et al. Pluronic P85-coated poly(butylcyanoacrylate) nanoparticles overcome phenytoin resistance in P-glycoprotein overexpressing rats with lithium-pilocarpine-induced chronic temporal lobe epilepsy. Biomaterials. 2016;97:110-121.

5. Duncan R. The dawning era of polymer therapeutics. Nat Rev Drug Discov. 2003;2(5):347-360.

6. Mahapatro A, Singh DK. Biodegradable nanoparticles are excellent vehicle for site directed in-vivo delivery of drugs and vaccines. J Nanobiotechnology. 2011;9:55.

7. Ramasamy T, Haidar ZS, Tran TH, et al. Layer-by-layer assembly of liposomal nanoparticles with PEGylated polyelectrolytes enhances systemic delivery of multiple anticancer drugs. Acta Biomater. 2014; 10(12):5116-5127.

8. Ramasamy T, Tran TH, Choi JY, et al. Layer-by-layer coated lipid-polymer hybrid nanoparticles designed for use in anticancer drug delivery. Carbohydr Polym. 2014;102:653-661.

9. Ramasamy T, Ruttala HB, Gupta B, et al. Smart chemistry-based nanosized drug delivery systems for systemic applications: A comprehensive review. J Control Release. 2017;258:226-253.
10. Ramasamy T, Ruttala HB, Chitrapriya N, et al. Engineering of cell microenvironment-responsive polypeptide nanovehicle coencapsulating a synergistic combination of small molecules for effective chemotherapy in solid tumors. Acta Biomater. 2017;48:131-143.

11. Dai T, Zhou S, Yin C, et al. Dextran-based fluorescent nanoprobes for sentinel lymph node mapping. Biomaterials. 2014;35(28):8227-8235.

12. Wang H, Dai T, Zhou S, et al. Self-Assembly Assisted Fabrication of Dextran-Based Nanohydrogels with Reduction-Cleavable Junctions for Applications as Efficient Drug Delivery Systems. Sci Rep. 2017; 7:40011.

13. Zhou S, Min X, Dou H, et al. Facile fabrication of dextran-based fluorescent nanogels as potential glucose sensors. Chem Commun. 2013; 49(82):9473-9475.

14. Huang L, Perrault C, Coelho-Martins J, et al. Induction of acquired drug resistance in endothelial cells and its involvement in anticancer therapy. J Hematol Oncol. 2013;6:49.

15. Pan T, Zhang M, Zhang F, et al. NDRG2 overexpression suppresses hepatoma cells survival during metabolic stress through disturbing the activation of fatty acid oxidation. Biochem Biophys Res Commun. 2017; 483(2):860-866.

16. Zhu D, Tao W, Zhang H, et al. Docetaxel (DTX)-loaded polydopaminemodified TPGS-PLA nanoparticles as a targeted drug delivery system for the treatment of liver cancer. Acta Biomater. 2016;30:144-154.

17. Dong LH, Cheng S, Zheng Z, et al. Histone deacetylase inhibitor potentiated the ability of MTOR inhibitor to induce autophagic cell death in Burkitt leukemia/lymphoma. J Hematol Oncol. 2013;6:53.

18. Yan ZX, Zheng Z, Xue W, et al. MicroRNA181a Is Overexpressed in T-Cell Leukemia/Lymphoma and Related to Chemoresistance. Biomed Res Int. 2015;2015:1-10.

19. Zhang Q, Li F. Combating P-glycoprotein-mediated multidrug resistance using therapeutic nanoparticles. Curr Pharm Des. 2013;19(37): 6655-6666.

20. Stella B, Peira E, Dianzani C, et al. Development and Characterization of Solid Lipid Nanoparticles Loaded with a Highly Active Doxorubicin Derivative. Nanomaterials. 2018;8(2):E110.

21. Peira E, Chirio D, Battaglia L, et al. Solid lipid nanoparticles carrying lipophilic derivatives of doxorubicin: preparation, characterization, and in vitro cytotoxicity studies. J Microencapsul. 2016;33(4):381-390.

22. Daga M, Ullio C, Argenziano M, et al. GSH-targeted nanosponges increase doxorubicin-induced toxicity "in vitro" and "in vivo" in cancer cells with high antioxidant defenses. Free Radic Biol Med. 2016;97: 24-37.

23. Noh YW, Kong SH, Choi DY, et al. Near-infrared emitting polymer nanogels for efficient sentinel lymph node mapping. ACS Nano. 2012; 6(9):7820-7831.

24. Sundaramoorthy P, Ramasamy T, Mishra SK, et al. Engineering of caveolae-specific self-micellizing anticancer lipid nanoparticles to enhance the chemotherapeutic efficacy of oxaliplatin in colorectal cancer cells. Acta Biomater. 2016;42:220-231.

25. Lemarchand $C$, Gref R, Passirani C, et al. Influence of polysaccharide coating on the interactions of nanoparticles with biological systems. Biomaterials. 2006;27(1):108-118.

26. Mu L, Feng SS. Vitamin E TPGS used as emulsifier in the solvent evaporation/extraction technique for fabrication of polymeric nanospheres for controlled release of paclitaxel (Taxol). J Control Release. 2002;80(1-3):129-144.

27. Liao J, Wang C, Wang Y, Luo F, Qian Z. Recent advances in formation, properties, and applications of polymersomes. Curr Pharm Des. 2012; 18(23):3432-3441. 
International Journal of Nanomedicine

Dovepress

\section{Publish your work in this journal}

The International Journal of Nanomedicine is an international, peerreviewed journal focusing on the application of nanotechnology in diagnostics, therapeutics, and drug delivery systems throughou the biomedical field. This journal is indexed on PubMed Central, MedLine, CAS, SciSearch ${ }^{\circledR}$, Current Contents ${ }^{\circledR} /$ Clinical Medicine,
Journal Citation Reports/Science Edition, EMBase, Scopus and the Elsevier Bibliographic databases. The manuscript management system is completely online and includes a very quick and fair peer-review system, which is all easy to use. Visit http://www.dovepress.com/ testimonials.php to read real quotes from published authors.

Submit your manuscript here: http://www.dovepress.com/international-journal-of-nanomedicine-journal 